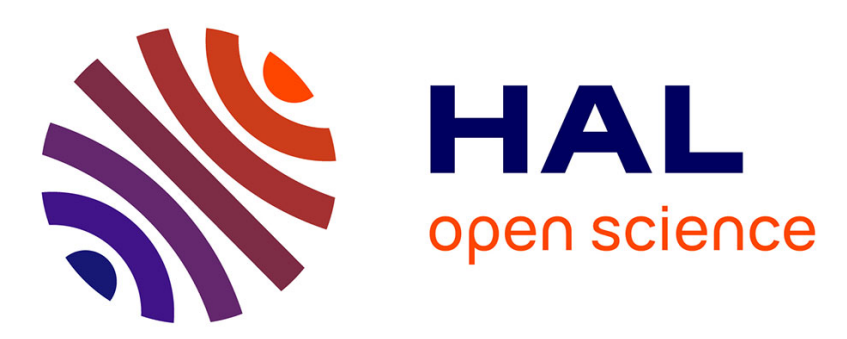

\title{
Potential feedback control for data scheduling in LTE cellular networks
}

\author{
Hamed Samie, Emmanuel Moulay, Patrick Coirault, Rodolphe Vauzelle, \\ Frédéric Launay
}

\section{- To cite this version:}

Hamed Samie, Emmanuel Moulay, Patrick Coirault, Rodolphe Vauzelle, Frédéric Launay. Potential feedback control for data scheduling in LTE cellular networks. 8th International Conference on Information, Intelligence, Systems \& Applications (IISA), Aug 2017, Larnaca, Cyprus. pp.1-6, 10.1109/IISA.2017.8316362 . hal-01735499

\section{HAL Id: hal-01735499 \\ https://hal.science/hal-01735499}

Submitted on 3 Feb 2021

HAL is a multi-disciplinary open access archive for the deposit and dissemination of scientific research documents, whether they are published or not. The documents may come from teaching and research institutions in France or abroad, or from public or private research centers.
L'archive ouverte pluridisciplinaire HAL, est destinée au dépôt et à la diffusion de documents scientifiques de niveau recherche, publiés ou non, émanant des établissements d'enseignement et de recherche français ou étrangers, des laboratoires publics ou privés. 


\title{
Potential Feedback Control for Data Scheduling in LTE Cellular Networks
}

\author{
Hamed Samie*, Emmanuel Moulay ${ }^{\dagger}$, Patrick Coirault*, Rodolphe Vauzelle ${ }^{\dagger}$ and Frédéric Launay* \\ *LIAS (EA 6315), Université de Poitiers, 2 rue Pierre Brousse, 86073 Poitiers Cedex 9, France \\ †XLIM (UMR CNRS 7252), Université de Poitiers, 11 bd Marie et Pierre Curie, \\ 86962 Futuroscope Chasseneuil Cedex, France, \\ Email: \{hamed.samie, emmanuel.moulay, patrick.coirault, rodolphe.vauzelle, frederic.launay\}@univ-poitiers.fr
}

\begin{abstract}
This article deals with a new Quality of Service (QoS) aware Two-Level Scheduler (TLS) for real-time (RT) data flows in downlink transmission of Long Term Evolution (LTE) cellular networks. It consists of two distinct scheduler levels. At the upper-level scheduler, the Potential Feedback Controller (PFC) determines the quota of data to transmit in order to meet the delay constraints of RT data flows while, at the lower-lever scheduler, the Proportional Fair (PF) scheduler is used to reach a high fairness among Users (UEs). The PFC is well suited for the upper-level scheduler since it maintains the queue length as close as possible to zero which leads to a low Packet Loss Ratio (PLR). A comparison, by using LTE-Sim simulator, with wellknown QoS aware scheduling methods Frame Level Scheduler (FLS), Exponential (EXP)-rule and Logarithmic (LOG)-rule has been provided which shows that our proposed TLS outperforms the EXP-rule and LOG-rule scheduling methods and it slightly decreases the PLR of RT data flows compared to the FLS scheduling method while it significantly reduces the computation time.
\end{abstract}

\section{INTRODUCTION}

Since 2009, LTE is represented as the next generation broadband technology with a promising framework (high data rate, scalable bandwidth and high mobility support) compared to the $3 \mathrm{G}$ networks [1]. LTE supports all Internet Protocol (IP)-based communication services containing Voice-over-IP (VoIP), video streaming, online gaming, and transmission control protocol-based services such as emailing and data transferring [2]. According to Ericsson Mobility, in 2015, LTE has exceeded 1 billion worldwide subscribers and it will reach 4.3 billion subscribers by 2021 [3]. Therefore, optimizing LTE features is an essential subject of research in wireless communications.

In RT multimedia services, in order to meet the Quality of Experience (QoE) requirements, the end-to-end delay constraints should suit the human perception of interactivity requirements [4]. For example, for the VoIP data flow, a maximum of $100 \mathrm{~ms}$ delay should be considered for a good perceived quality [5]. As soon as the RT data flow decoding starts with the chosen playout delay, it is compulsory for every encoded packet to respect this deadline. Therefore, if the encoded packet does not arrive within this deadline, it will be considered as a lost packet [4]. As a result, in RT multimedia services, ensuring bounded delivery delays is equivalent to reducing packet losses.

LTE specifications inset a data bearer which identifies each data flow based on its QoS requirements [6]. The packet scheduler sorts packets of a given data bearer using a packet filter based on the well-known fivetuple: source and destination IP addresses, source and destination ports, protocol identifier [7]. Therefore, packet scheduler can assign available radio resources per-flow basis.

LTE standard does not inflict any specific scheduling method to vendors and gives them the freedom to design their own scheduling methods [7]. Consequently, the problem of defining a simple scheduling method for allocating available radio resources among UEs has extensively been studied. Classical scheduling methods such as Maximum Throughput (MT) [8], PF [9] and Weighted Round Robin [10] are not specified to meet the RT data flow QoS requirements. Therefore, several contributions propose QoS aware scheduling methods for RT data flows in downlink transmission of LTE cellular networks. A delay-prioritized scheduling method is proposed in [11]. In [12], a specific packet scheduler for VoIP data flow is proposed which firstly assigns all available radio resources to the VoIP data flows then, it assigns the remaining radio resources to the other data flows. In [13], Packet Prediction Mechanism (PPM) is proposed for downlink RT services which allocates available radio resources by predicting the behavior of future incoming packets. In [2], a scheduling algorithm for downlink multimedia streaming is proposed in order to reduce the Hard Handoff (HO) procedure effect when a UE is moving from one cell to another. The famous delay-sensitive scheduling methods EXP-rule and LOG-rule have been presented in [14]. In [4], a QoS aware Two-Level Scheduler (TLS) has been proposed which determines a quota of data to transmit in frame time scale at the upper-level scheduler by exploiting a discrete-time feedback control called FLS and at the lower-lever scheduler, the TLS uses the PF scheduler method in order to reach a high level of fairness among UEs. Finally, in [15], [16], based on the TLS proposed in [4], three level schedulers FLS-Advanced (FLSA) and FLSA - Cross Carrier (FLSA-CC) are proposed in order to have a better performance by prioritizing real-time traffics.

By applying the PFC first proposed in [17], [18], instead of the FLS at the upper-level scheduler, we develop a new QoS aware TLS for RT data flows in downlink transmission of LTE cellular networks. To demonstrate the efficiency of our proposed TLS, a realistic simulation has been provided by using the LTE-Sim simulator [19]. The performance of 
our proposed TLS is compared with respect to the EXP-rule, the LOG-rule and the FLS scheduling methods. The results confirm that our proposed TLS is able to respect the QoS requirements of RT data flows while it decreases significantly the computation time.

The paper is organized as follows: in Section (II), an overview on packet scheduling in LTE networks is provided. In Section (III), we develop our QoS aware TLS for RT data flows in downlink LTE systems. Section (IV) reports the simulation results. Finally, a conclusion is addressed in (V).

\section{Overview of PACKet Scheduling in LTE}

In LTE, radio resources are allotted into the time-frequency domain [20]. In the time domain, they are distributed among UEs every Transmission Time Interval (TTI) that lasts $1 \mathrm{~ms}$. Conversely, in the frequency domain, the total bandwidth is divided into $180 \mathrm{kHz}$ sub-channels where each sub-channel contains 12 successive and equally spaced Orthogonal FrequencyDivision Multiplexing (OFDM) sub-carriers. In LTE, time is split in frame scales where each frame is composed of 10 successive TTIs. Moreover, each TTI itself consists of two time slots with $0.5 \mathrm{~ms}$ length where each slot contains either six or seven OFDM symbols for extended or normal Cyclic Prefix (CP) length respectively [21]. In LTE, Physical Resource Block (PRB), which is a time-frequency radio resource containing one time slot $(0.5 \mathrm{~ms})$ and one frequency subchannel $(180 \mathrm{kHz})$, is the smallest unit of radio resource that can be assigned to a UE [20].

Radio Resource Management (RRM) system has been designed to improve the usage of limited available radio resources. RRM exploits a mix of Media Access Control (MAC) and Physical (PHY) layers functions such as: Packet Scheduler (PS); Channel Quality Indicator (CQI) reporting; link adaptation through Adaptive Modulation and Coding (AMC) and Hybrid Automatic Re-transmission Request (HARQ) [7]. At the base station (eNodeB), the PS is the responsible for distributing radio resources between UEs. In our work, we are interested in the PS module performance in the downlink direction. Figure 1 represents the main RRM modules which interact with the PS in the downlink direction.

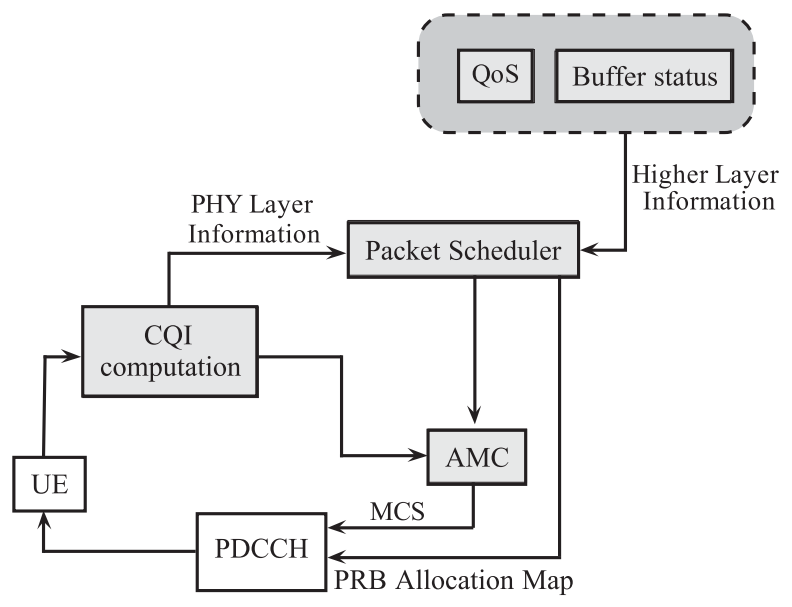

Fig. 1: A simple model of packet scheduler
The entire process of radio resource scheduling is represented by a sequence of operations which are repeated every TTI [7]:

1) Each active UE computes the CQI by decoding a pilot signal and send it back to the eNodeB on the Physical Downlink Control Channel (PDCCH).

2) Based on the CQI information and QoS constraints, the PS allocates available radio resources to active UEs.

3) The AMC module chooses the best modulation schemes (i.e., QPSK, 16-QAM or 64-QAM) for data transmission by scheduled UEs.

4) The scheduling information (scheduled UEs, allocated PRBs and selected modulation schemes) are sent to UEs on the PDCCH.

5) Each scheduled UE decodes its information data to the proper Physical Downlink Shared Channel (PDSCH).

In [4], the TLS has been proposed in order to meet the packet delay constraints of the RT data flows in LTE downlink direction. The TLS is composed of two distinct levels that interact together to dynamically allocate available radio resources between the UEs. Figure 2 illustrates the TLS design for the RT data flows in the LTE downlink direction. At the upper-level scheduler, the FLS determines for each frame (10 TTIs) the amount of data of the $i$-th RT data flow $u_{i}(n)$ to transmit in order to meet its packet delay constraints. The non-RT data flows like best effort flows will be scheduled after transmitting the quota defined by the upper-level scheduler due to their longer packet delay. It should be mentioned that the upper-level scheduler does not take into account the quality of the transmission channel. On the other hand, the lowerlevel scheduler, every TTI, allocates the available PRBs to UEs by exploiting the PF scheduling algorithm in order to reach a high level of fairness between multimedia flows [22]. The lower-level scheduler should take into account the quota of the data to transmit within the current frame defined by the upper-level scheduler for the RT data flows. Finally, if the lower-level scheduler meets this quota before starting a new frame, the best effort data flow is scheduled using the PF scheduling algorithm (see Figure 2). We refer the reader to [4] for more information on the TLS design.

\section{PFC FOR UPPER-LEVEL SCHEDULER}

In this section, by exploiting the PFC first proposed in [17], [18], instead of the FLS at the upper-level scheduler, we develop a novel TLS for RT data flows in downlink transmission of LTE cellular networks in order to decrease the PLR of RT data flows and the computation time compared to the TLS given in [4] (see Figure 3).

Let $t_{n, i}$ be the starting time of the $n$-th frame for the $i$-th RT data flow. Thus, the sampling interval $\Delta t(n)=t_{n+1, i}-t_{n, i}$ corresponds to the LTE frame legnth. According to [4], evolution of the $i$-th RT data flow queue length can be represented by the following equation:

$$
q_{i}(n+1)=q_{i}(n)+d_{i}(n)-u_{i}(n)
$$

where $q_{i}(n)$ is the $i$-th queue length at $t_{n, i} ; q_{i}(n+1)$ is the $i$-th queue length at $t_{n+1, i} ; u_{i}(n)$ is the amount of data 


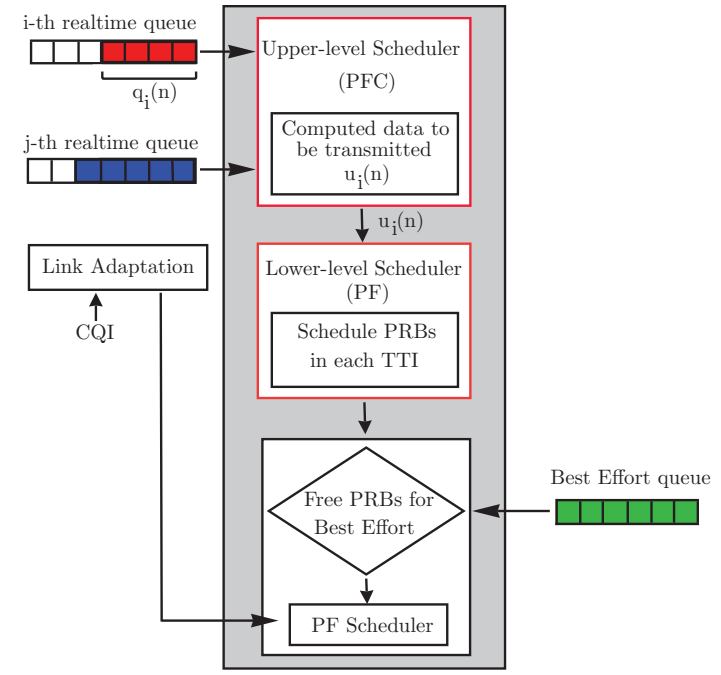

Fig. 2: Two-Level scheduler for real-time data flow in downlink direction.

to transmit during the $n$-th frame; $0 \leq d_{i}(n) \leq d_{M}$ is the amount of the data to be filled in the queue during the $n$-th frame.

The potential control has originally been applied in robotics to avoid collisions with obstacles [23], [24]. The PFC is well suited to the upper-level scheduling problem because it maintains the state of the system (queue length) higher than a target value (queue $\min _{\text {m }}=0$ ) while keeping the state as close as possible to this target value in order to optimize the PLR for the RT data flows in downlink transmission. Beside the performance improvement, by applying the PFC at the upper-level scheduler, we can take advantage of its simple stabilization approach and its short computation time compared to the FLS proposed in [4].

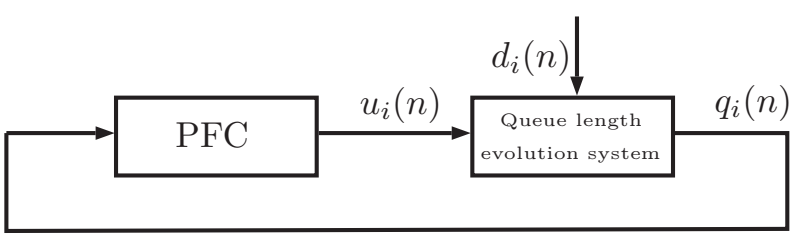

Fig. 3: Feedback control loop for the upper-level scheduler

Let us review the PFC for system (1).

Theorem 3.1: System (1) under the initial condition $q_{i}(0)>$ $c$ with $c \leq 0$, with the feedback control

$$
u_{i}(n)=-k_{1}\left(q_{i}(n)-c\right)-\frac{k_{2}}{q_{i}(n)-c}
$$

where $u(n)=0$ for $n<0$ and $-1<k_{1}<0$ and $k_{2}>$ 0 is asymptotically stable with respect to the attractive and invariant set

$$
S=\left[2 \sqrt{k_{2}\left(k_{1}+1\right)} ; \frac{d_{M}\left(1-2 k_{1}\right)+\alpha+\sqrt{\left(d_{M}\left(1-2 k_{1}\right)+\alpha\right)^{2}+8 k_{1}^{2} k_{2}}}{4 k_{1}^{2}}\right]
$$

where $\alpha=\sqrt{d_{M}^{2}-4 k_{1} k_{2}}-\sqrt{4 k_{1} k_{2}}$. Furthermore, we have

$$
q_{i}(n) \geq 2 \sqrt{k_{2}\left(k_{1}+1\right)}+c
$$

for all $n \in \mathbb{N}^{*}=\mathbb{N} \backslash\{0\}$.

The proof of the Theorem 3.1 is provided in [17, Theorem 1].

\section{Simulations}

In this section, we provide a realistic simulation of a multi-cell LTE Wireless Cellular Network (WCN) in order to compare the performance of our proposed TLS with respect to the one given in [4], and well-known scheduling strategies LOG-rule and Exp-rule [25]. For our simulations, we used the LTE-Sim simulator [19] which is an open-source simulator for LTE networks.

Our multi-cell WCN simulation scenario consists of 19 cells where we have one eNodeB and a variable number of UEs in each cell (see Figure 4).

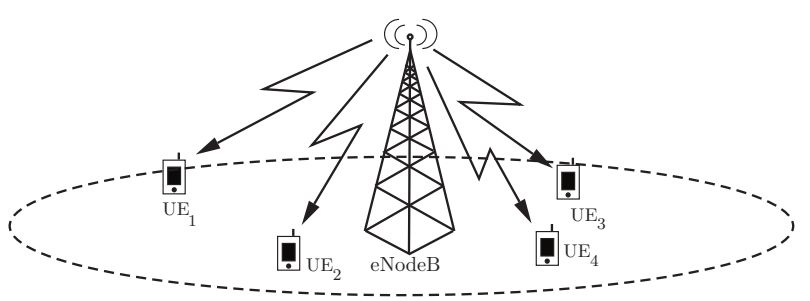

Fig. 4: Simulation scenario for a cell

Mobility of each UE is described by the random way-point model [26]. A $10 \mathrm{MHz}$ downlink bandwidth is considered for each base station. We have considered a variable mobility speed in order to analyze both pedestrian and vehicular UEs. Furthermore, the impact of the inter-cell interference is also considered. Table I provides a list of the simulation parameters. It should be mentioned that all simulation results are averaged over four simulations.

TABLE I: Simulation parameters

\begin{tabular}{|l|c|}
\hline Simulation time & $100 \mathrm{~s}$ \\
\hline Number of cells & 19 \\
\hline Radius of each cell & $0.5 \mathrm{~km}$ \\
\hline Bandwidth of each cell & $10 \mathrm{MHz}$ \\
\hline Number of UEs per cell & $10,15,20$ \\
\hline Speed of UEs & $\begin{array}{l}3 \mathrm{~km} / \mathrm{h} \text { (pedestrian), } \\
120 \mathrm{~km} / \mathrm{h} \text { (vehicular) }\end{array}$ \\
\hline
\end{tabular}

In our simulations, each UE receives one video flow, one VoIP flow and one best effort flow at the same time. The video flow is encoded with $H .264$ standard compression at $128 \mathrm{kbps}$ average rate. The VoIP flow has adopted an ON/OFF Markov model where during the ON period, every $20 \mathrm{~ms}$, the resource sends 20 bytes sized packets (source data rate is $8 \mathrm{kbps}$ ) while during the OFF period the rate is zero because of the presence of a voice activity detector. At last, we considered infinite buffer sources for the best effort flow [19]. At the Physical layer, we have supposed that the eNodeB exploits two antenna ports and a power transmission equal to $43 \mathrm{dbm}$, uniformly diffused over all the 50 available sub-channels. In our simulations, the urban channel model is considered 


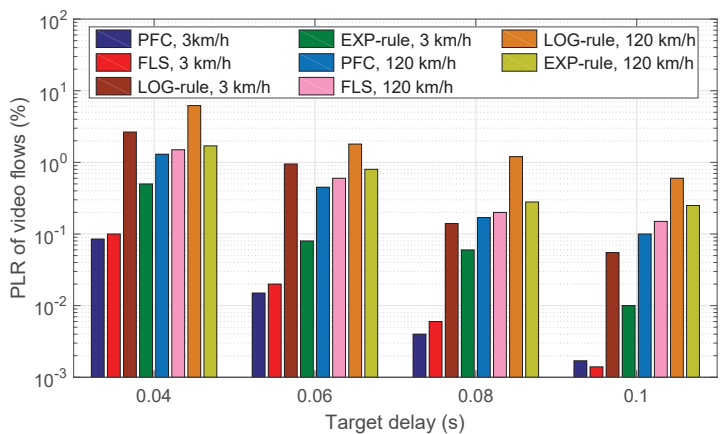

(a) 10 UEs

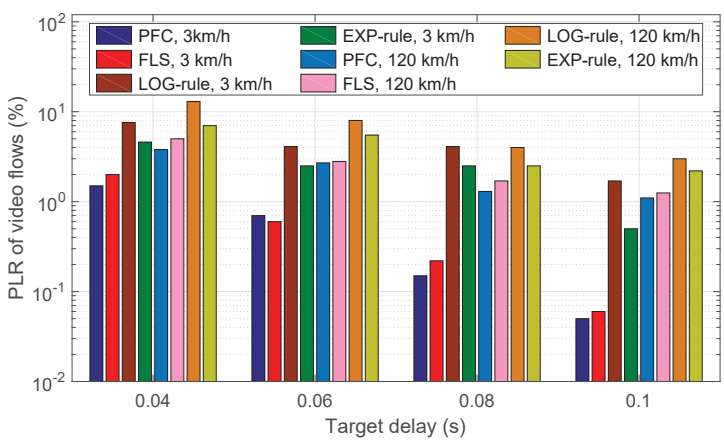

(b) 15 UEs

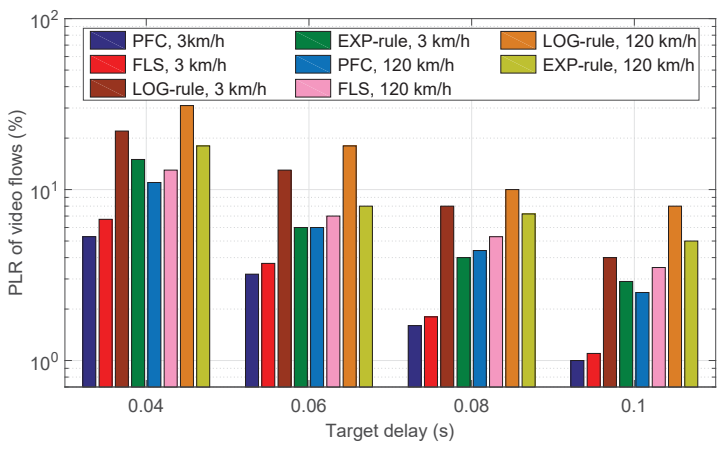

(c) 20 UEs

Fig. 5: Packet Loss Ratio of video flows

where the Path-Loss (PL) between the eNodeB and the UE is estimated according to [27] by

$$
P L=128.1+37.6 \log (d)
$$

where $d$ is the distance between the UE and the eNodeB in $\mathrm{km}$. The large scale shadowing fading is modeled via a log-normal distribution and finally, time-frequency correlated signal multipath is modeled by using the Rayleigh fading channel model [19].

According to Theorem 3.1, in order to maintain the $q_{i}(n) \geq$ 0 , we must have $-1<k_{1}<0$ and $k_{2}>0$. A numerical computation shows that by taking $k_{1}$ closer to -1 we have the $q_{i}(n)$ closer to 0 . Therefore, we choose $k_{1}=-0.9$ and $k_{2}=0.5$ for our simulations. Also, we choose $c=0$.

In our simulations, we considered a variable set of delay targets in the interval [40ms - $100 \mathrm{~ms}]$. It should be mentioned that we considered the same target delays for both video and VoIP flows to ensure that they are synchronously played out

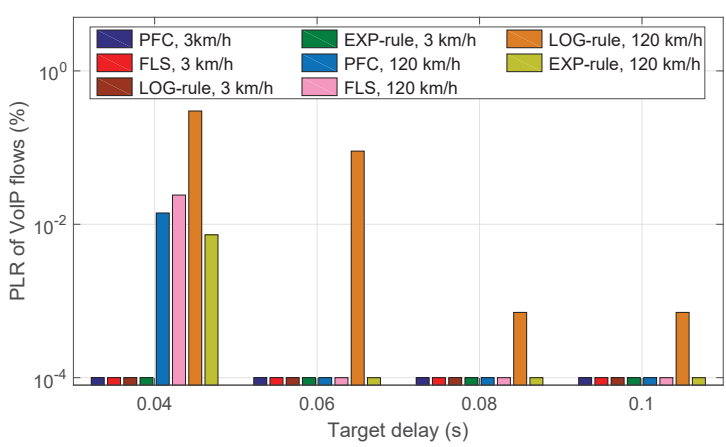

(a) 10 UEs

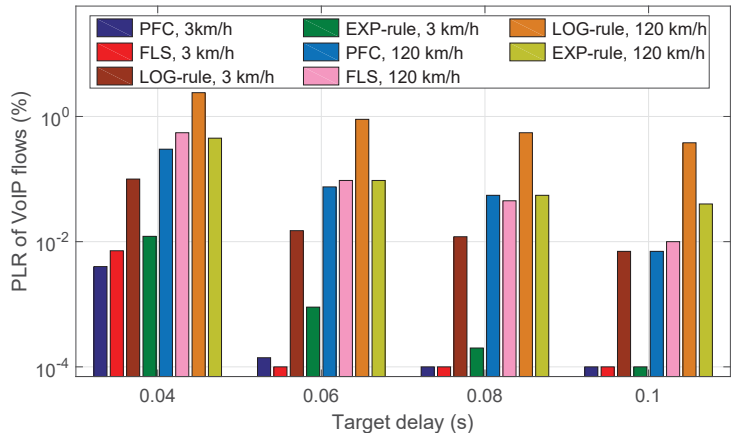

(b) 15 UEs

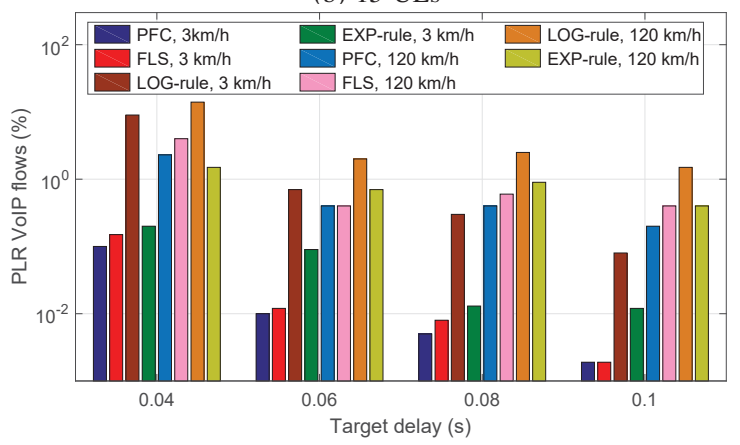

(c) 20 UEs

Fig. 6: Packet Loss Ratio of VoIP flows

at the same UE, which is an important characteristic in video conferencing [4]. It is important to remark that the enqueued packets are deleted from the transmission queue just when they expire or on the other word, they have not been transmitted before their target delay. In multimedia communications, packets received by the UE after their target delay have to be considered as lost packets because they are no longer usable by the decoding process.

Concerning multimedia data flows, PLR is a standard metric for evaluating QoS offered by the system at network layer. As the best effort data flows do not have strict QoS specifications, we consider the fairness index and the total goodput to compare the performance of different scheduling algorithms [4].

Figures 5 illustrates the achieved PLR of video flows. It is easy to see that the achieved PLR increases with a higher network load (number of UEs). Furthermore, network with $120 \mathrm{~km} / \mathrm{h}$ user mobility speed achieves a higher PLR 


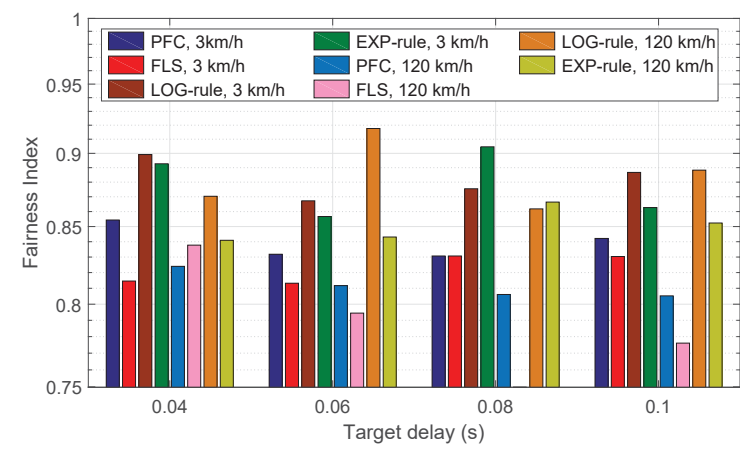

(a) 10 UEs

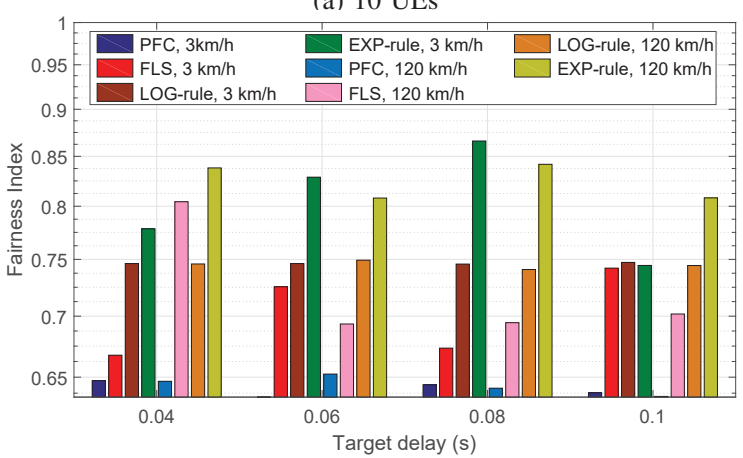

(b) 15 UEs

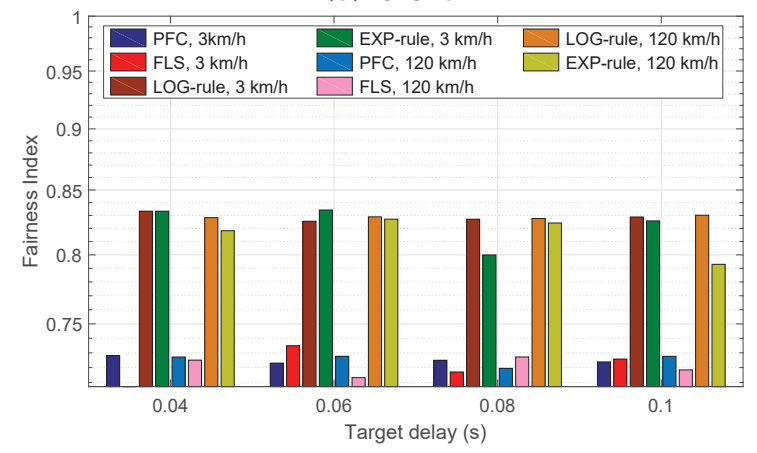

(c) 20 UEs

Fig. 7: Fairness Index of best effort flows.

compared to the one with $3 \mathrm{~km} / \mathrm{h}$ user mobility speed. This is due to the fact that the transmission channel quality varies faster with a higher mobility speed which leads to a higher slip in MCS selection by AMC module. It is important to mention that the achieved PLR increases by taking a lower target delay due to a larger quota of packets passing the deadline determined by the QoS requirements. Consequently, we have the highest PLR for the simulation scenario of a network with 20 UEs per cell, $40 \mathrm{~ms}$ the target delay and $120 \mathrm{~km} / \mathrm{h}$ user mobility speed.

Figures 6 illustrates the achieved PLR of VoIP flows. The achieved PLR for VoIP flows is considerably lower compared to the achieved PLR of video flows. As we have the PF scheduling method at the lower-level scheduler, the VoIP flows have the higher priority due to their lower source bit rate. The PFC and FLS have better performances compared to the LOGrule and EXP-rule in maintaining the target delay of the video

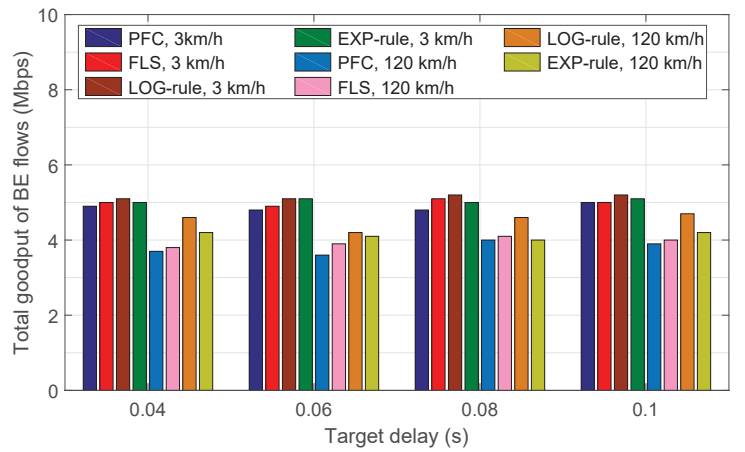

(a) 10 UEs

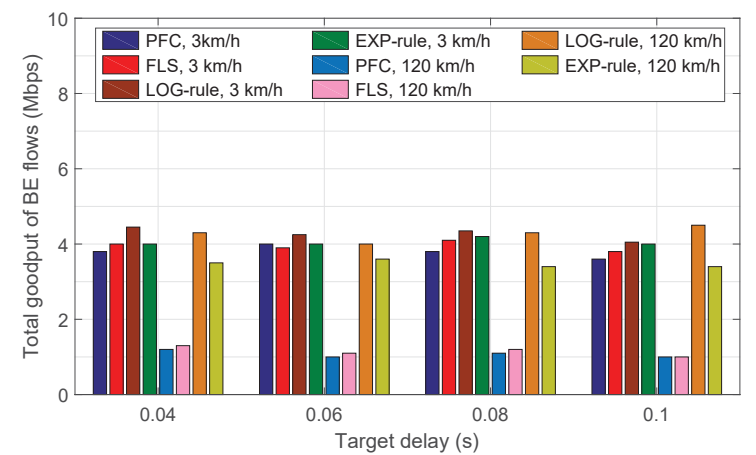

(b) 15 UEs

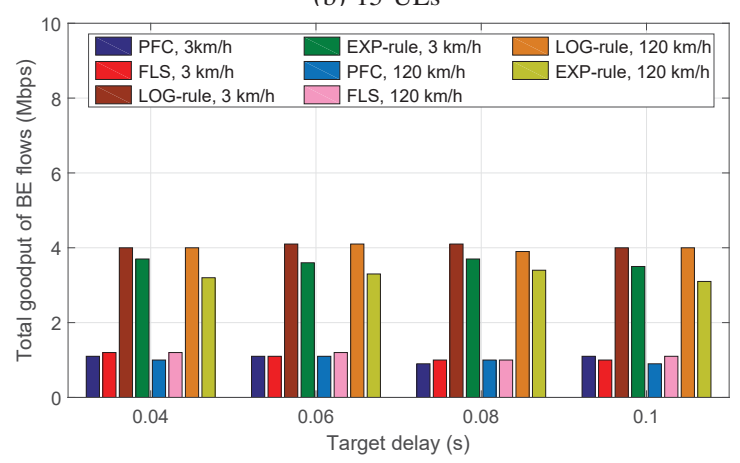

(c) 20 UEs

Fig. 8: Total Goodput of best effort flows.

and VoIP flows while the PFC has a slightly lower achieved PLR of the video and VoIP flows with respect to the FLS.

Figures 7 shows the fairness index for different number of UEs with different mobility speeds. It is easy to see that all considered scheduling methods provide a high degree of fairness. Figures 8 provides the total goodput of BE flows for different number of UEs with different mobility speeds. The goodput is determined as the rate of useful bits which has been transmitted successfully by BE flows. It is easy to see that the goodput decreases with a higher network load. It should be mentioned that we have a higher goodput with respect to the PFC and FLS by using the LOG-rule and EXP-rule scheduling methods. This is due to the fact that in TLS (with the PFC or the FLS) the RT data flows have a higher priority compared to the non-RT data flows (i.e, BE flows).

Finally, Figure 9 provides the computation time of the PFC and the FLS for a 10s simulation for a video data flow with 
0.08s target delay. Obviously, the FLS has a significant higher computation time with respect to the PFC. According to [4], for each flow, the FLS should execute $(M-1)$ multiplications and $3(M-1)+1$ sums in order to compute $u_{i}(n)$ where $M$ is the target delay of the RT data flow.

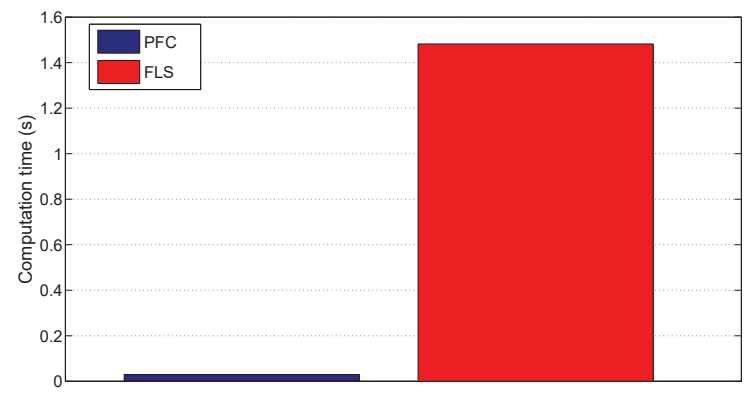

Fig. 9: Computation time of the FLS and the PFC.

\section{CONCLUSION}

In this article, we developed a new QoS aware TLS for RT data flows in downlink transmission of LTE cellular networks. The proposed TLS exploits the PFC at the upper-level scheduler. The PFC is well suited to the upper-level scheduling problem because it maintains the state of the system (queue length) higher than a target value (queue $\min =0$ ) while keeping the state as close as possible to this target value in order to optimize the PLR for the RT data flows in downlink transmission. Simulation results show that by using our proposed TLS, UEs experience a lower PLR specially for video flows compared to the EXP-rule and the LOGrule scheduling methods. Moreover, our proposed TLS slightly improves the PLR for RT data flows compared to the FLS while it significantly reduces the computation time.

\section{ACKNOWLEDGEMENTS}

This work is supported by the region Poitou-Charentes of France and The French National Center for Scientific Research (CNRS).

\section{REFERENCES}

[1] M. Rumney et al., LTE and the Evolution to $4 G$ Wireless: Design and Measurement Challenges. John Wiley \& Sons, 2013.

[2] Q. Liu and C. W. Chen, "Smart Downlink Scheduling for Multimedia Streaming Over LTE Networks With Hard Handoff," IEEE Transactions on Circuits and Systems for Video Technology, vol. 25, no. 11, pp. 1815$1829,2015$.

[3] Ericsson AB, "Ericsson Mobility Report: On the pulse of the networked society," Tech. Rep., June 2016, https://www.ericsson.com/res/docs/ 2016/ericsson-mobility-report-2016.pdf.

[4] G. Piro, L. A. Grieco, G. Boggia, R. Fortuna, and P. Camarda, "Twolevel downlink scheduling for real-time multimedia services in LTE networks," IEEE Transactions on Multimedia, vol. 13, no. 5, pp. 1052 1065, 2011.

[5] S. Na and S. Yoo, "Allowable propagation delay for VoIP calls of acceptable quality," in Advanced Internet Services and Applications. Springer, 2002, pp. 47-55.

[6] H. Ekstrom, "QoS control in the 3GPP evolved packet system," IEEE Communications Magazine, vol. 47, no. 2, pp. 76-83, 2009.
[7] F. Capozzi, G. Piro, L. A. Grieco, G. Boggia, and P. Camarda, "Downlink packet scheduling in LTE cellular networks: Key design issues and a survey," IEEE Communications Surveys \& Tutorials, vol. 15, no. 2, pp. $678-700,2013$.

[8] S. Schwarz, C. Mehlführer, and M. Rupp, "Low complexity approximate maximum throughput scheduling for LTE," in 44th Asilomar Conference on Signals, Systems and Computers, 2010, pp. 1563-1569.

[9] H. Kim, K. Kim, Y. Han, and S. Yun, "A proportional fair scheduling for multicarrier transmission systems," in IEEE 60th Vehicular Technology Conference, 2004, pp. 409-413.

[10] H. Luo, S. Ci, D. Wu, J. Wu, and H. Tang, "Quality-driven cross-layer optimized video delivery over LTE," IEEE Communications Magazine, vol. 48, no. 2, 2010.

[11] K. Sandrasegaran, H. A. M. Ramli, and R. Basukala, "Delay-prioritized scheduling (DPS) for real time traffic in 3GPP LTE system," in IEEE Wireless Communications and Networking Conference, 2010, pp. 1-6.

[12] S. Choi, K. Jun, Y. Shin, S. Kang, and B. Choi, "MAC scheduling scheme for VoIP traffic service in 3G LTE," in IEEE 66th Vehicular Technology Conference, 2007, pp. 1441-1445.

[13] W. K. Lai and C.-L. Tang, "QoS-aware downlink packet scheduling for LTE networks," Computer Networks, vol. 57, no. 7, pp. 1689-1698, 2013.

[14] B. Sadiq, S. J. Baek, and G. De Veciana, "Delay-optimal opportunistic scheduling and approximations: The log rule," IEEE/ACM Transactions on Networking, vol. 19, no. 2, pp. 405-418, 2011.

[15] E. Skondras, A. Michalas, A. Sgora, and D. D. Vergados, "A downlink scheduler supporting real time services in LTE cellular networks," in 6th IEEE International Conference on Information, Intelligence, Systems and Applications (IISA), 2015, pp. 1-6.

[16] _ "Q "QoS-aware scheduling in LTE-A networks with SDN control," in 7th IEEE International Conference on Information, Intelligence, Systems and Applications (IISA), 2016, pp. 1-6.

[17] D.-D. Phan, E. Moulay, P. Coirault, F. Launay, and P. Combeau, "Potential feedback control for the power control in LTE," IEEE Transactions on Automatic Control, vol. 60, no. 9, pp. 2506-2511, 2015.

[18] D. D. Phan, E. Moulay, P. Coirault, A.-M. Poussard, and R. Vauzelle, "Potential feedback control for the power control in wireless sensor networks," IET Control Theory \& Applications, vol. 9, no. 13, pp. 2022 2028, 2015.

[19] G. Piro, L. A. Grieco, G. Boggia, F. Capozzi, and P. Camarda, "Simulating LTE cellular systems: an open-source framework," IEEE Transactions on Vehicular Technology, vol. 60, no. 2, pp. 498-513, 2011.

[20] 3GPP, "Evolved Universal Terrestrial Radio Access (E-UTRA); Physical Channel and Modulation (Release 13), 3GPP TS 36.211," Tech. Rep.

[21] S. Sesia, I. Toufik, and M. Baker, LTE-the UMTS long term evolution. Wiley Online Library, 2015.

[22] E. Dahlman, S. Parkvall, J. Skold, and P. Beming, 3G evolution: HSPA and LTE for mobile broadband. Academic Press, 2010.

[23] P. Ogren, E. Fiorelli, and N. E. Leonard, "Cooperative control of mobile sensor networks: Adaptive gradient climbing in a distributed environment," IEEE Transactions on Automatic Control, vol. 49, no. 8, pp. 1292-1302, 2004.

[24] E. Rimon and D. E. Koditschek, "Exact robot navigation using artificial potential functions," IEEE Transactions on Robotics and Automation, vol. 8, no. 5, pp. 501-518, 1992.

[25] B. Sadiq, R. Madan, and A. Sampath, "Downlink Scheduling for Multiclass Traffic in LTE," Journal on Wireless Communications and Networking, 2009.

[26] T. Camp, J. Boleng, and V. Davies, "A survey of mobility models for ad hoc network research," Wireless Communications and Mobile Computing, vol. 2, no. 5, pp. 483-502, 2002.

[27] 3GPP, "Technical Specification Group Radio Access Network; Physical layer aspects for evolved Universal Terrestrial Radio Access (UTRA) (Release 7), 3GPP TS 25.814," Tech. Rep. 